\title{
Design of Automatic Vision-based Inspection System for Monitoring in an Olive Oil Bottling Line
}

\author{
Slim ABDELHEDI \\ Commande de Machines \\ Electriques et Réseaux de \\ Puissance (CMERP) \\ Tunisia
}

\author{
Khaled TAOUIL \\ Commande de Machines \\ Electriques et Réseaux de \\ Puissance (CMERP) \\ Tunisia
}

\author{
Bassem HADJKACEM \\ Commande de Machines \\ Electriques et Réseaux de \\ Puissance (CMERP) \\ Tunisia
}

\begin{abstract}
Automated vision inspection has become a vital part of the quality monitoring process. This paper compares the development and performance of two methodologies for a machine vision inspection system online for high speed conveyor. The first method developed is the Thresholding technique image processing algorithms and the second method is based on the edge detection. A case study was conducted to benchmark these two methods.

Special effort has been put in the design of the defect detection algorithms to reach two main objectives: accurate feature extraction and on-line capabilities, both considering robustness and low processing time. An on-line implementation to inspect bottles is reported using new communication technique with GigE Vision camera and industrial Gigabit Ethernet network.

The system is validated on olive oil bed. The implementation of our algorithm results in an effective real-time object tracking. The validity of the approach is illustrated by the presentation of experiment results obtained using the methods described in this paper.
\end{abstract}

\section{Keywords}

GigE vision camera, Image processing, Quality Monitoring, Defects detection.

\section{INTRODUCTION}

In thin strip perforation processes speed can reach values that exceed $1 \mathrm{~m} / \mathrm{s}$. To monitor product quality several parameters should be checked, using different measuring tools in many cases. Manual inspection process could be much slower than the production process leading to less frequent inspection. Any deviation in quality parameters between inspection runs will result in scrap production.

The fact that machine vision systems are noncontact, fast and can easily be computerized make them a potential tool for online quality monitoring during actual production in such processes. Automated Visual Inspection (AVI) [12] can estimate dimensions of work parts using visual information and is most often automated by employing machine vision techniques.

Machine vision is a tool used to control products in automated production lines in different fields such as food industry [13]. Such a tool has the advantage of non-contact measurements without running off the production process.

Vision systems have been applied for many applications including inspection of surface defects $[11,18]$, for Bottle inspection [2, 11, 12], for pharmaceutical tablets [15], for
Press Part Sorting [16] and for Potatoes Defects Detection [17].

Using multiple image sensors at different levels of the production line and different angles of shots allows monitoring the different stages of the production process and collection of the information needed to assess product quality and flow of production (See figure 1) $[1,2,3,4]$.

GigE Vision is a gigabit Ethernet based interface adapted to machine vision [5]. GigE vision cameras [19] use gigabit Ethernet and therefore can be fitted within the open structure of the industrial Gigabit Ethernet network [6, 7, 14]. This allows the transmission of images and controls signals between cameras and control systems at high speeds over long cable lengths.

Therefore, we can consider the design of real time machine vision applications based on the Ethernet architecture. In this paper we present a GigE Vision system used for quality monitoring in an olive oil bottling line. This system is seamlessly integrated within the production network and allows the detection of defaults in cap and oil level. The detection of anomalies is transferred to the control system via discrete alarms on the industrial Gigabit Ethernet network. This system is mainly composed of a camera for taking pictures of products on the production line, special lighting to highlight faults, computer visualization and image processing related to alarm generation and automatic ejection [2].

In this paper, an attempt is made to evaluate the performance of the traditional image processing algorithms $[5,8]$ in a online machine vision Inspection system. Comparison is based on the processing time, accuracy, preprocessing time, and orientation requirement. Results of the evaluation are presented and discussed.

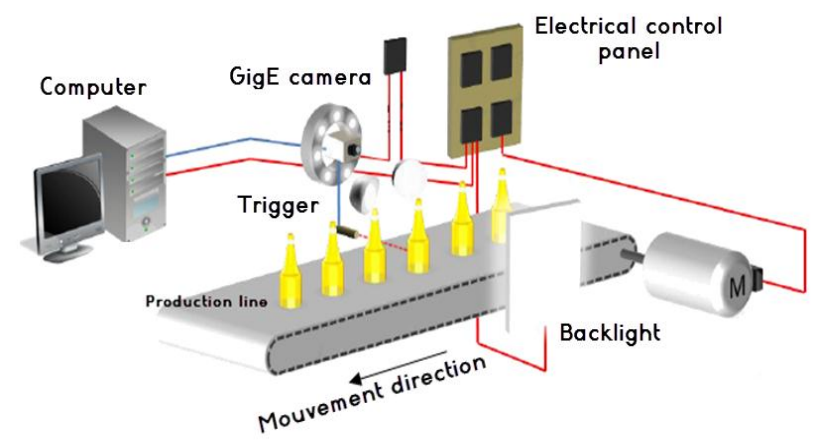

Fig 1: Machine Vision System 


\section{SYSTEM HARDWARE DESCRIPTION}

Figure 1 shows the complete system setup. A part moving on the conveyor belt is sensed by the proximity sensor.

The sensor sends a signal to the computer with which it is interfaced. The camera which is interfaced with the same computer is external triggered with signal and captures the image of the bottle. The obtained image is then processed by the processing software installed on the computer and then the Pass/Fail decision is made.

This whole operation is done in few milliseconds depending upon the sensor delay timing, speed of the camera and the processing speed of the computer. The necessary hardware components are:

\subsection{Camera CCD and optics}

An imaging sensor with an excellent noise performance is required by this application, which is more important for video applications. CCDs are superior to Complementary Metal Oxide Semiconductor (CMOS) image sensors, as far as SNR and dynamic range are concerned. In this way, CCD image sensor is selected for this application. The scrolling cadence of the bottles, so the time to shoot is decisive. Indeed, it must be short enough that the object appears fixed.

The technological solution exists for some manufacturers offering industrial digital cameras equipped for some of CCD high sensitivity, allowing a fast exposure time and producing good quality images (See figure 2) [5].

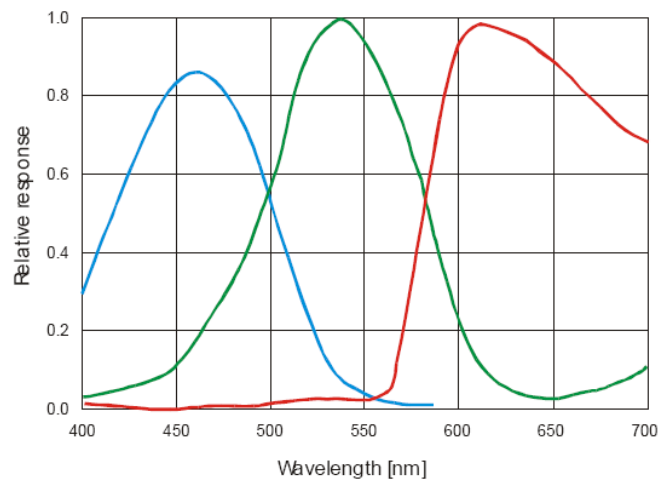

(b) Responce spectal

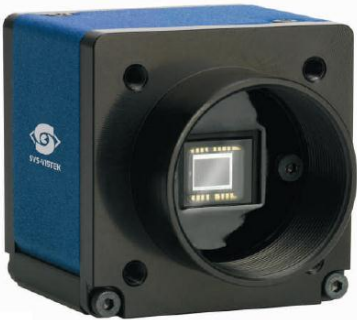

(a) Camera SVCam-ECO Line eco424

\section{Fig 2: Camera SVCam-ECO Line eco424, sensor CCD ICX 424 1/3"'Interline, 640 x 480 Pixel and responce spectal.}

We use an industrial CCD color (Bayer Pattern) camera. It is a progressive (full frame) camera. It is GigE vision and GenICam standard compliant. This digital camera doesn't need a frame grabber but a Gigabit Ethernet network adapter is needed to ensure working with high data rate [7].

Table I: Performances required

\begin{tabular}{|l|l|}
\hline \multicolumn{2}{|c|}{ Camera settings } \\
\hline Resolution & $640 \times 480$ \\
Sensor size & $1 / 3 "$ \\
Pixel size & $7.4 \mu \mathrm{m} \times 7.4 \mu \mathrm{m}$ \\
Exposure time & $3 \mu \mathrm{s}-2 \mathrm{~s}$ \\
Frame rate & $124 \mathrm{fps}$ \\
\hline
\end{tabular}

\subsubsection{Calculation of Field of View:}

Field of View (FOV) is defined as the viewable area of the object under inspection or the portion of the object that fills the camera's sensor.

$$
\text { Field of view }=(\mathrm{Dr}+\mathrm{Lm})(1+\mathrm{Pa})
$$

Where $\mathrm{Dr}$ is the required field of view, $\mathrm{Lm}$ is the maximum variation in part location and orientation and $\mathrm{Pa}$ is the allowance for camera pointing as a percentage.

Then, Field of view $=(96 \mathrm{~mm}+10 \mathrm{~mm})(1+10 \%)=116.6 \mathrm{~mm}$.

The factor by which the FOV should be expanded depends on the skill of the people who maintain the vision system. A value of $10 \%$ is a common choice. In order to fill the monitor with the feature of interest more clearly, the FOV of $120 \mathrm{~mm}$ is selected.

\subsubsection{Focal Length:}

The focal length is a lens' pivotal parameter. To represent an object completely on the CCD chip, one should calculate the focal length for the object height and width.

Focal Length of the Width $=\left(\mathrm{WD} * \mathrm{CCD}_{\mathrm{Width}}\right) /\left(\mathrm{S}_{\mathrm{obj}}+\right.$ $\mathrm{CCD}_{\text {Width }}$ )

Focal Length of the Height $=\left(\mathrm{WD} * \mathrm{CCD}_{\text {Height }}\right) /\left(\mathrm{S}_{\mathrm{obj}}+\right.$ CCD Height)

Where WD is Working Distance and $\mathrm{S}_{\mathrm{obj}}$ is Size Of Object.

So,

Focal Length of Width $=(300 \mathrm{~mm} * 4.8 \mathrm{~mm}) /(96 \mathrm{~mm}+$ $4.8 \mathrm{~mm})=14.285 \mathrm{~mm}$

To adjust focal lengths, Zoom Lens is necessary. Due to some disadvantages of Zoom lens such as weight, size and Price Fixed focal length lens whose focal length is lower than the calculated one can be used. Especially in the case of small objects, the working distance may be smaller than the selected lenses minimal working distance (MOD). In this case, extension rings is placed between the lens and the camera to decrease MOD.

\subsubsection{Exposure time:}

This is the time during which light is creating an exposure. To hold image blur to within one pixel, the exposure time must be, $\mathrm{Te}=\mathrm{FOV} /\left(\mathrm{V}_{\mathrm{p}} * \mathrm{~N}_{\mathrm{p}}\right)$

Where Te is the exposure time, $V_{p}$ is the velocity of the conveyor and $\mathrm{N}_{\mathrm{p}}$ is the number of pixels in horizontal direction.

Then, $\mathrm{Te}=116.6 /(1000 * 640)=0.00018$ seconds. This exposure can be achieved with electronic shutter or strobed illumination. 


\subsubsection{Image blur:}

In this application, there will be image blur since the bottles are traveling in the conveyor line. Generally, Blurred images are caused by poor lens quality and incorrect lens setup. The rate of part motion, the size of FOV, and the exposure time are have influence on the magnitude of the image blur.

$\mathrm{B}=\mathrm{V}_{\mathrm{p}} * \mathrm{~T}_{\mathrm{e}} * \mathrm{~N}_{\mathrm{p}} / \mathrm{FOV}$

Where $\mathrm{B}$ is the blur in pixels. The actual blur for this application can be calculated as:

$\mathrm{B}=1000 * 0.00018 * 640 / 116.6=0.9879$ pixels.

\subsubsection{Cycle time}

Cycle time is defined as the delay between two consecutive image acquisitions. Let $\mathrm{d}$ be the distance between two consecutive bottles an $\mathrm{V}$ the scrolling speed, $\mathrm{t}_{\text {cycle }}$ is given by the following expression:

$$
\mathrm{T}_{\text {cycle }}=\mathrm{d} / \mathrm{V}
$$

For $\mathrm{d}=10 \mathrm{~cm}$ and $\mathrm{V}=1 \mathrm{~m} / \mathrm{s}$, we have $\mathrm{t}_{\text {cycle }}=0.1 \mathrm{~s}$; that's a 10 images/second cadence. The used camera allows for up to 124 frames per second. (Figure 3)

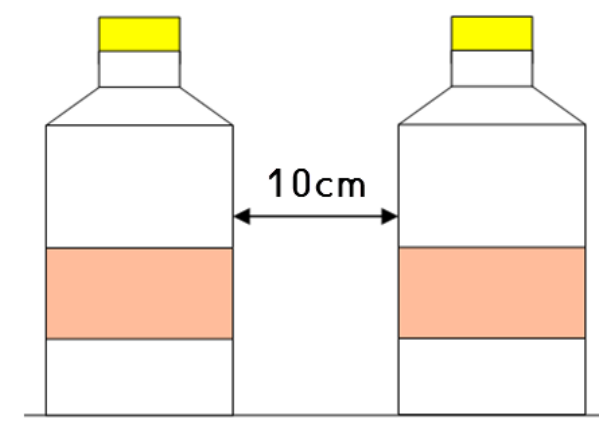

Fig 3: Distance between two consecutive bottles

\subsection{Communication Protocol}

GigE vision standard is based on UDP/IP [2, 5]. It consists of four major parts. The first part defines a mechanism that allows applications to detect and enumerate devices and defines how devices obtain a valid IP address. The second part defines a GigE vision control protocol (GVCP) that allows the configuration of detected devices and guarantees transmission reliability. The third part defines a GigE vision streaming protocol (GVSP) that allows applications to receive information from devices. The last part defines bootstrap registers that describe the device itself (e.g., current IP address, serial number, manufacturer information, etc.).

For a peer-to-peer connection of a GigE camera to a PC a network address assignment based on LLA (Local Link Address) is recommended. This involves a network mask "255.255.0.0" as well as a fixed first part "169.254.xxx.xxx" of the network address range. A GigE camera will fall back to LLA soon after recognizing that no DHCP server is available and that no fixed network address was assigned to the camera.

GigE Vision offers many features which make it quite suitable for an image capturing interface in high speed robot vision systems.

GigE Vision provides enough bandwidth to transmit video in fast frame way which is an important issue for high speed vision systems. For this reasons, we believe that GigE vision is a suitable interface for high speed industrial inspection[5, 26].

\subsection{Lighting}

The choice of the lighting is essential to the quality and pertinence of acquired image. The complexity of the subsequent processing depends on the contrast level between objects and background. In our case we used two kinds of lighting sources positioned at both sides of the observed objects. The first one is a direct ring sunlight directly mounted around the camera.

This kind of lighting provides a huge amount of light within the exposure space. The radial shaped lighting reduces shadows allowing for good definition of the object's color. We also use a polarizing filter to help reduce brilliance surfaces. The second is a backlight which helps improve the perception of the cork shape and liquid level (See figure 4 and 5) $[4,29]$.


Fig 5: 2-D arrayed LED panel

\subsection{Part sensor}

Often in the form of a light barrier or sensor, this device sends a trigger signal when it senses that a part is in close proximity [25]. The sensor shown in figure 6 tells the machine vision system when a part is in the correct position for an image to be acquired. The specifications are:

- Rated supply voltage: $12 . . .24$ V DC with reverse polarity protection

- Switching capacity in mA:100 mA, overload and short circuit protection

- Delay response: $<2 \mathrm{~ms}$

- Maximum sensing distance: $0.4 \mathrm{~m}$ diffuse

As it is only required to sense the bottle, a proximity sensor which can sense any object within $15 \mathrm{~cm}$ will serve the purpose. 


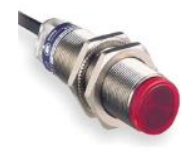

Fig 6: Photo-electric sensor-XUB-multi-Sn 0..18m12..24 VDC-Cable 2m

\section{SYSTEM SOFTWARE DESCRIPTION}

We use MATLAB 7.12b for the system software development. Image acquisition toolbox is used to capture the images from the GigE vision camera interfaced [27, 28] with the PC. The image processing toolbox is used to develop the algorithm to inspect the bottle [3,6]. The Instrumentation Control Toolbox is used to read the proximity sensor signals. Image of a bottle is captured using the GigE vision camera as shown in figure 7 . The simple thresholding algorithm is applied.

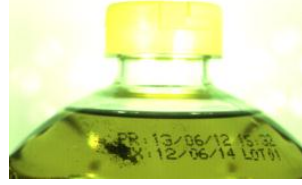
Off-line image

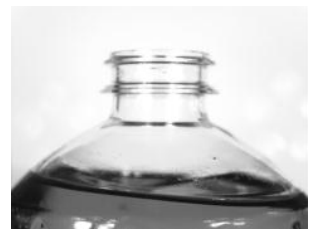

(c) On-line line gray level image: No cap and under fill level (a) Original image:

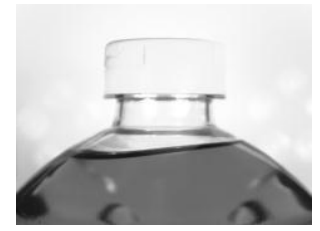

(b) On-line gray level image: Normal cap and good level

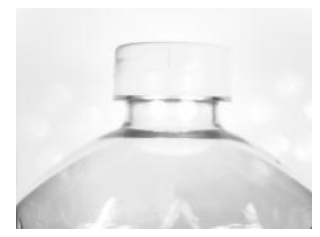

(d) On-line gray level image: Normal cap and under fill level

\section{Fig 7: Capturing images}

\section{DEFECT INSPECTION METHODS}

\subsection{Threshold techniques}

\subsubsection{Description}

We use a hardware based triggering method. The acquisition, analysis and decision are made in real time from images acquired by the industrial camera VGA and transmitted through the interface GigE Vision. The shooting is triggered by a sensing system directly connected to the camera.

There are many image processing techniques useful for machine vision inspection. These techniques involve histogram analysis, boundary detection, region growing and others. The choice of the techniques is primarily based on the types of inspection and application. In this work, we are used a local thresholding algorithms [20], edge detection [9] and area calculation. All these techniques need to be performed in a binary image, which only contains black and white pixels.

In thresholding, the color-image or gray-scale image is reduced to a binary image. Thresholding is a process of converting a grayscale input image to a bi-level image by using an optimal threshold. The purpose of thresholding is to extract those pixels from some image which represent an object.

Though the information is binary the pixels represent a range of intensities. For a thresholding algorithm to be really effective, it should preserve logical and semantic content.

There are two types of thresholding algorithms:

- Global thresholding algorithms

- Local or adaptive thresholding algorithms

In global thresholding, a single threshold for all the image pixels is used. When the pixel values of the components and that of background are fairly consistent in their respective values over the entire image, global thresholding could be used. In adaptive thresholding, different threshold values for different local areas are used.

In our paper adaptive thresholding is used and was given as follow:

1) Calculate the histogram of RGB image.

2) Calculate the histogram of Blue color intensity in the range $[0,255]$.

3) Compute the threshold (T) that separated the bottle and background in original image by using the Otsu method [9].

Figure 8 shows an intensity histogram of the original image.

The horizontal axis presents the intensity value of the bottle image ranged from 0 to 255 , while the vertical axis represents the pixel probability of corresponding intensity value.

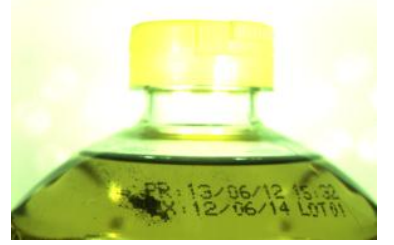

(a) Original image: Off-line image



(b) $R, G$ and $B$ values of original image

Fig 8: $R, G$ and $B$ values of the image

In the area calculation, the region of the image is the portion with the boundary, where the boundary is represented by those pixels that have both black and white neighbors. The application of this technique is to calculate the total number of the black pixels that contain the region and the boundary of the image.

An inspection threshold value is predetermined and implemented in the algorithm to distinguish the good from the 
defective parts. The area of good parts should be very close to each other and is always greater than a defective part [8].

\subsubsection{Image processing flowchart}

To reduce the processing time, we extract a first region of interest ROI1 to inspect the cap and a second region of interest ROI2 for inspection of liquid level.

This flowchart explains the approach work in this paper and the various parts of our detection algorithm for cap closure and level content (See figure 9).
The detection steps are described:

(1) The sensor detects presence of a bottle.

(2) The camera load the image processing.

(3) Extract Regions Of Interests ROI1 and ROI2.

(4) Calculate areas thresholds.

(5) Compare values sought to areas thresholds.

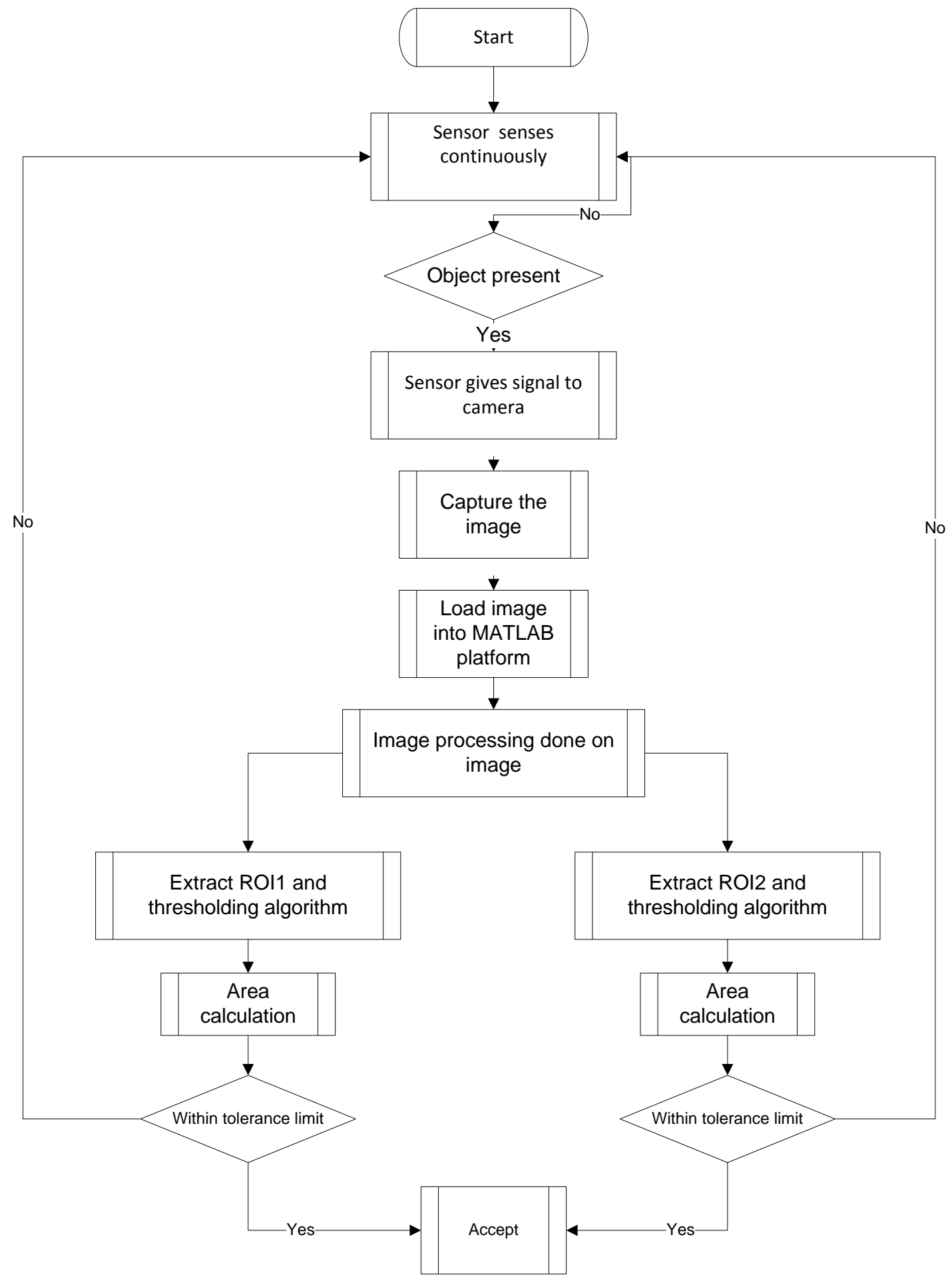

Fig 9: Diagram for detect cap and level content 


\subsection{Edge detection techniques}

Image of a filled bottle is captured using a GigE vision camera as shown in Figure 7. The image is cropped to make it a normalized image with respect to height of the conveyor belt. For this paper, Canny Edge detection technique [21, 22] can be applied. The required steps for edge detection algorithm are given in Tables II:

\section{Table II: CANNY ALGORITHM}

\begin{tabular}{|c|l|}
\hline $\mathbf{N}^{\circ}$ & STEPS \\
\hline 1 & Read the image I. \\
\hline 2 & Convolve a 1D Gaussian mask with I. \\
\hline 3 & $\begin{array}{l}\text { Create a 1D mask for the first derivative of the } \\
\text { Gaussian in the x and y directions. }\end{array}$ \\
\hline 4 & $\begin{array}{l}\text { Convolve I with G along the rows to obtain Ix, } \\
\text { and down the columns to obtain Iy. }\end{array}$ \\
\hline 5 & $\begin{array}{l}\text { Convolve Ix with Gx to have Ix', and Iy with Gy } \\
\text { to have Iy'. }\end{array}$ \\
\hline 6 & $\begin{array}{l}\text { Find the magnitude of the result at each pixel } \\
(\mathrm{x}, \mathrm{y}) .\end{array}$ \\
$\qquad M(x, y)=\sqrt{I_{x}^{\prime}(x, y)^{2}+I_{y}^{\prime}(x, y)^{2}}$ \\
\hline
\end{tabular}

\subsection{Speed performance measurement}

In this paper, we evaluate the performance of our algorithm and the limits of the vision system with two conveyor speeds using the thresholding technique and Canny edge detection.

The experimental results are shown in figures 10 and 11:

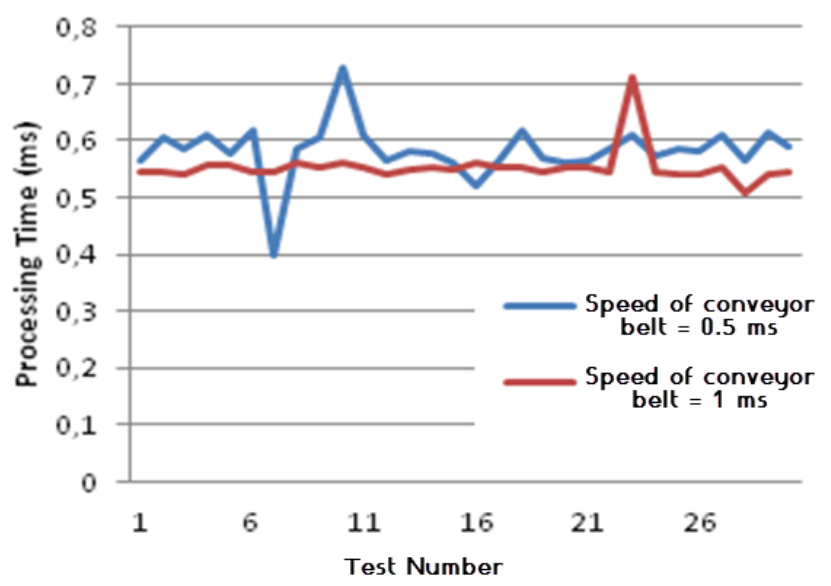

Fig 10: Diagram for detect cap and level content user thresholding technique

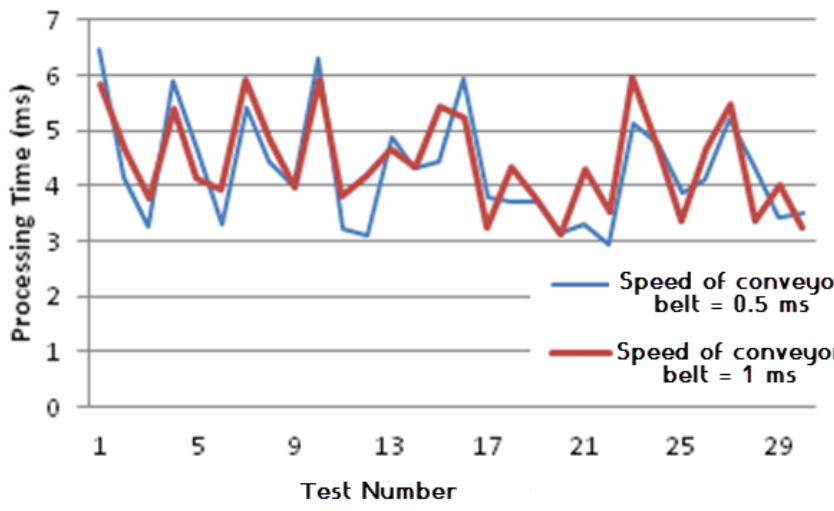

Fig 11: Diagram for detect cap closure and level content user edge detection
According to the measurements found, we see that the thresholding method is more rapid and adapted to real time inspection.

\section{RESULTS}

\subsection{Defect Inspection with thresholding approach}

After applying the proposed approach, we measure level of liquid contents and detect cap closure we need to find two regions of interest (ROIs) (See figure 12).

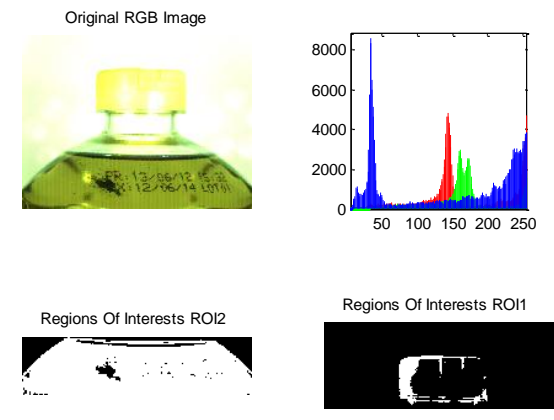

Fig 12: Regions of interests: ROI1 and ROI2

There are different situation for cap and level. All of them are demonstrated in figure 13. Oil bottle is rejected from the production line, if the two conditions are satisfied for the level and the cap.

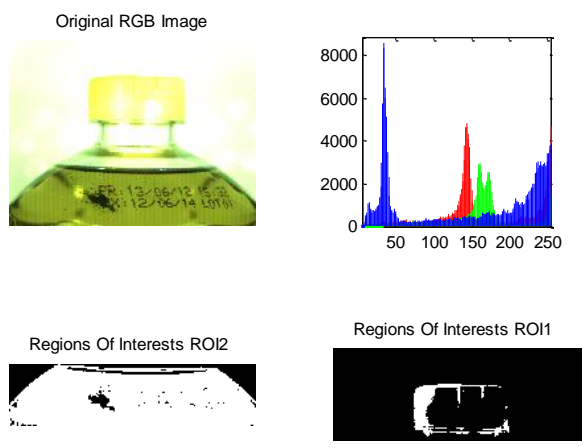

(a) Normal cap and good level

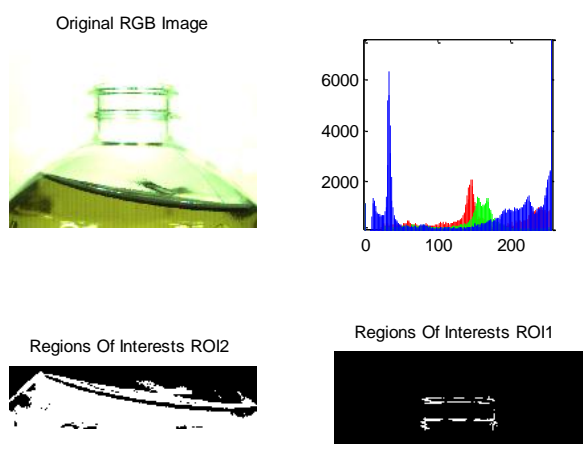

(b) No cap and under fill level 



Regions Of Interests ROI1

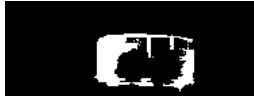

(c) Normal cap and under fill level

Fig 13: Yellow color thresholding with Backlight

\subsection{Defect Inspection with edge detection approach}

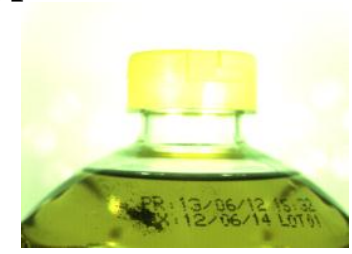

(a) Original image: Off-line image



(c) Regions of interests: ROI1 and ROI2

Fig 14: Regions of interests and image profile



Fig 15: Profile detection line
In the case where the cap is present, the profile line of ROI 1 is presented in Figure 15. Two peaks at the ROI indicate the presence of the cap.

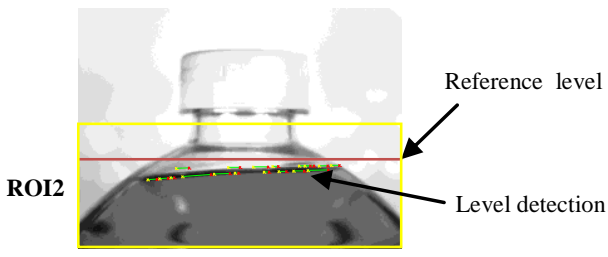

Fig 16: Level detection line

For liquid level detection, Hough transform is used [23, 24]. This technique detects straight line segments in a given binary image. The problem of determining the location and orientation of straight lines in images arises in many diverse areas of image processing and computer vision. The required steps for Hough transform algorithm are given in Tables III:

\section{Table III: HOUGH TRANSFORM ALGORITHM}

\begin{tabular}{|c|l|}
\hline $\mathbf{N}^{\circ}$ & STEPS \\
\hline 1 & Read the image I. \\
\hline 2 & Find the edges in the image \\
\hline 3 & For all pixels in the image \\
\hline 4 & If the pixel(x,y) is an edge \\
\hline 5 & For all the theta angles \\
\hline 6 & $\begin{array}{c}\text { Calculate rho for the pixel(x,y) and the angle (theta) } \\
\text { Increment that position (rho,theta) in the accumulator }\end{array}$ \\
\hline 7 & Show the hough space \\
\hline 8 & Find the highest values in the accumulator \\
\hline 9 & draw the line with the highest value in the input image \\
\hline
\end{tabular}

\section{CONCLUSION}

In this paper we describe the different steps for dimensioning a machine vision system .The GigE vision is used to insure seamless integration within the production line. Image processing, fault detection and alarm generation are done on a common workstation. The use of well defined shooting conditions allowed for simplified image processing technique for cap detection and liquid level verification with sufficient precision to qualify for real time production monitoring within the bottling chain.

\section{REFERENCES}

[1] Yao Rong, Dong He, "Rapid Detection Method for Fabric Defects Based on Machine Vision", 2010 International Conference on Computer Application and System Modeling (ICCASM 2010), p 662-666.

[2] Mohammad A. Younes, S. Darwish, "Online Quality Monitoring of Perforated Steel Strips Using an Automated Visual Inspection (AVI) System", Proceedings of the 2011 IEEE ICQR, p 575-579.

[3] R. Siricharoenchai ,W. Sinthupinyo," Using Efficient Discriminative Algorithm in Automated Visual Inspection of Feed", Communication Software and Networks (ICCSN), 2011 IEEE, p 95-99.

[4] Che-Seung Cho, Byeong-Mook Chung, "Development of Real-Time Vision-Based Fabric Inspection System", IEEE TRANSACTIONS ON INDUSTRIAL ELECTRONICS, VOL. 52, NO. 4, AUGUST 2005

[5] E. Norouznezhad (2008), "A HIGH RESOLUTION SMART CAMERA WITH GIGE VISION 
EXTENSION FOR SURVEILLANCE APPLICATIONS", ICDSC 2008. Second ACM/IEEE International Conference on Distributed Smart Cameras (ICDSC'08).

[6] "The Elements of GigE Vision", whitepaper, Basler Vision Technologies, http://www.baslerweb.com/

[7] "Digital Gigabit Ethernet Area-Scan Camera", Version 1.6, SVSVISTEC,http://www.jm-vistec.com

[8] WANG Hongzhi (2008), "An Improved Image Segmentation Algorithm Based on Otsu Method", International Symposium on Photoelectronic Detection and Imaging 2007: Related Technologies and Applications, edited by Liwei Zhou, Proc. of SPIE Vol. $6625,66250 \mathrm{I}$.

[9] A. Soni, M. Aghera, "Machine Vision Based Part Inspection System Using Canny Edge detection Technique", Proc. of the 4th International Conference on Advances in Mechanical Engineering, September 23-25, 2010, S.V. National Institute of Technology, Surat - 395 007, Gujarat, India.

[10] L. Yazdi, A. S. Prabuwono, "Feature Extraction Algorithm for Fill Level and Cap Inspection in Bottling Machine", 2011 International Conference on Pattern Analysis and Intelligent Robotics 28-29 June 2011, Putrajaya, Malaysia.

[11] F. Duan, Y. Wang, and H. Lin, "A Real-time Machine Vision System for Bottle Finish Inspection," in Proc. 8th International Conference on Control, Automation, Robotics and Vision (IEEE), 2004.

[12] P. Kumar, "A Roadmap for Designing an Automated Visual Inspection System”, (C2010 International Journal of Computer Applications (0975 - 8887) Volume 1 - No. 19.

[13] Khaled TAOUIL (2008), "Machine Vision Based Quality Monitoring in Olive Oil Conditioning", Image Processing Theory, Tools and Applications, 2008. IPTA 2008. First Workshops on.

[14] GenICam Standard Specification, Version 1.0, http://www.genicam.org/

[15] Miha Možina and al., "Real-time image segmentation for visual inspection of pharmaceutical tablets", Machine Vision and Applications (2011) 22:145-156

[16] Habibullah Akbar, Anton Satria Prabuwono, "The Design and Development of Automated Visual Inspection System for Press Part Sorting", Computer Science and Information Technology, 2008. ICCSIT '08. International Conference on , p 683-686.

[17] J. Jin and al., "Methodology for Potatoes Defects Detection with Computer Vision", ISBN 978-952-572602-2 (Print), 978-952-5726-03-9 (CD-ROM) Proceedings of the 2009 International Symposium on I nformation
Processing (ISIP'09) Huangshan, P. R. China, August 21-23, 2009, pp. 346-351.

[18] M. Islam, R. Sahriar and B. Hossain, “An Enhanced Automatic Surface and Structural Flaw Inspection and Categorization using Image Processing Both for Flat and Textured Ceramic Tiles", International Journal of Computer Applications (0975 - 888) Volume 48- No.3, June 2012.

[19] W. He, K. Yuan, H. Xiao and Z. Xu, "A high speed robot vision system with GigE Vision Extension", Proceedings of the 2011 IEEE International Conference on Mechatronics and Automation August 7 - 10, Beijing, China.

[20] H. K. Singh, S. K. Tomar and P. K. Maurya, "Thresholding Techniques applied for Segmentation of RGB and multispectral images", Proceedings published by International Journal of Computer Applications ${ }^{\circledR}$ (IJCA)ISSN: 0975 - 8887

[21] K. J Pithadiya, C.K Modi and J. D Chauhan, "Comparison of optimal edge detection algorithms for liquid level inspection in bottles", Second International Conference on Emerging Trends in Engineering and Technology, ICETET-09

[22] Canny, John, "A Computational Approach to Edge Detection," IEEE Transactions on Pattern Analysis and Machine Intelligence,Vol. PAMI-8, No. 6, 1986, pp. 679-698.

[23] C. Di Ruberto, "Generalized Hough Transform for Shape Matching", International Journal of Computer Applications (0975 - 8887) Volume 48- No.1, June 2012

[24] Kumar D and V. Prasad G, "Image Processing Techniques to Recognize and Classify Bottle Articles", National Conference on Advances in Computer Science and Applications with International Journal of Computer Applications (NCACSA 2012) Proceedings published in International Journal of Computer Applications ${ }^{\circledR}$ (IJCA)

[25] "Shedding Light on Machine Vision", Machine VIsion Lighting, whitepaper,http://www.crossco.com

[26] Whitepaper, Basler Vision Technologies, http://www.baslerweb.com/

[27] "GenICam - the new Programming Interface Standard for Cameras",whitepaper, Basler Vision Technologies, http://www.baslerweb.com/

[28] "Digital Gigabit Ethernet Area-Scan Camera", Version 1.6, SVSVISTEC,http://www.jm-vistec.com

[29] Ajay Kumar, "Vision-based Fabric Defect Detection: A Survey", Indian Institute of Technology Delhi, Hauz Khas, New Delhi - 110016, India. 